\title{
Koniocortex-Like Network Application to Business Intelligence
}

\author{
J. Fombellida
}

D. Andina

\begin{abstract}
Koniocortex-Like Network model is a Bio-Inspired Neural Network structure that tries to replicate the architecture and properties of the biological koniocortex section of the brain. The structure is composed by different kinds of artificial neurons that interplay between them to create a competitive model that can be used to classify patterns. The classification performance obtained is based on different properties like lateral inhibition, metaplasticity and intrinsic plasticity, that allows a natural evolution of the network until obtaining the desired results. This kind of network has been applied to synthetic and real data showing big potential, now the network capabilities are tested using other state-of-the-art real data application: the classification of credit data from the Australian Credit Approval Database.
\end{abstract}

Index Terms-Metaplasticity, Koniocortex, KLN, Business Intelligence, ACAD, Feature Extraction, Competition

\section{INTRODUCTION}

The human brain presents a region inside the cerebral cortex in the form of a granular layer (layer IV) that it is called koniocortex. This brain area contains abundance of spiny stellae neurons that gives this layer its granular texture, these neurons are directly connected to the thalamus. The thalamus is the main relay station from the senses to the cortex and provide neural projections to the spiny neurons of the koniocortex.

The Koniocortex-Like Network (KLN) is an Artificial Neural Network (ANN) model based on an unsupervised learning paradigm, that is composed by two layers that try to replicate the behavior of its biological counterpart. The first layer contains neurons similar to the thalamo-cortical neurons of the thalamus, and the second layer include the neurons that resemble the spiny and inhibitory interneurons that form the biological koniocortex. This two-layered structure constitute a network capable of classifying patterns using its competition and auto-organization properties.

According to [1] the real koniocortex can be considered as a competitive network due to the fact that only a small number of spiny neurons are active when the thalamus connection provides sensory stimuli to this region. This is similar to the Winners Takes All (WTA) paradigm that is included in many unsupervised network models so the biological structure and properties have been used as inspiration for this new artificial model. The main difference between the classic ANN approach for the WTA and the KLN is that while conventional competitive neurons detects the most active neuron using external calculations, in the case of the KLN the winning neuron emerges naturally from the interaction between neurons inside the network dynamics. In addition the non-winning neurons are silenced due to the activity of its neighbor neurons, not because they are algorithmically reset.

Considering the KLN model the main properties involved in competitive learning are synaptic metaplasticity and intrinsic plasticity. Intrinsic plasticity adjusts the global excitability of the neuron so that highly excited neurons will be less excitable in the future, and vice versa. This article is in line with the works presented previously [2], [3], [4], [5], [6], [7]. The KLN is applied to the classification of the patterns included in the Australian Credit Approval Database (ACAD) [8]. The results are evaluated considering the following performance figures: sensitivity, specificity and accuracy, and the validation is made using the 10-fold cross validation method. The paper is organized as follows. Section 2 presents the Business Intelligence (BI) concept and the application of bio-inspired systems to its specific problems. Section 3 presents a detailed description of the database and the algorithms. In section 4 the experimental results obtained are shown, a brief discussion of these results is included and a comparison with other state-ofthe-art algorithms is presented. Finally, section 5 summarizes the main conclusions.

\section{BUSINESS INTELLIGENCE AND ANN APPLICATION}

Business Intelligence (BI) solutions applied to business services and utilities is considered as one of the principal possibilities inside the development of what it is called Industry 4.0 revolution. System networks need powerful intelligence systems to process all the available information coming from the different users to study their behavior so services and business results can be improved based on the existing knowledge embedded on Business Data (BD).

The constant evolution on technology research provide new solutions that introduce new technical and organizational concepts. Companies are switching to IT-driven efficient management of complex information [9], [10], that performs an added value processing of the information coming from the different actors. This processing improves efficiency, reliability 
and economy. New business processes are being established to accommodate these technologies and market evolutions.

BI allows collecting, correlating and analyzing events from multiple sources by taking advantage from existing tools while gaining end-to-end management of the entire network. BI is the practice of interpreting the data to make useful businessoriented decisions. BI decision support applications facilitate this multi-dimensional analysis like online analytical processing, click-stream analysis, balance score-card, preparation, visualization, querying, reporting, charting, data mining for text content and voice, forecasting, geospatial analysis, enterprise portal implementation, knowledge management, digital dashboard access and other cross-functional activities.

According to [11] $\mathrm{BI}$ requires tools and technologies focused on enhanced decision making. The results obtained are normally used in supply chains, sales, finances and marking. BI generates reliable decisions that reduces the risks associated to the inconsistencies in the classic decision-making process [12]. So BI systems are well recognized as important contributors for decision-making. BI systems are most commonly identified as technological solutions holding quality information in well-designed data stores, enabling them to make the right decisions or take the right actions.

BD and BI systems complexity make their evaluation difficult. Due to the multi-disciplinary aspects involved in these systems, a classic engineering approach may not provide a successful adequate solution [13]. To tackle the complex problem of providing $\mathrm{BI}$ solutions based on $\mathrm{BD}$, bio-inspired ANNs have to be considered, because these systems have the potential to provide a more efficient and effective use of explicit and implicit knowledge present in the BD.

This is the case that we are presenting in this article, a novel structure and learning algorithm inspired in different biological properties like metaplasticity and intrinsic plasticity. These basic characteristics of the neural connections are believe to be crucial in achieving the biological Deep Learning that allows biological brains to successfully deal with realworld complex problems. In the experiments that have been performed in the frame of this investigation, the KLN method is applied to the classification of the ACAD and the resulting system achieve performance that is comparable with the most powerful methods of the state-of-the-art with the additional advantage that being an unsupervised learning algorithm there is no need of previous information about the classification result of the patterns.

\section{MATERIAL AND METHODS}

\section{A. Credit Scoring Method}

The financial crisis has put the focus of investigation in banking issues, specially in the ones related to the approval of credits. Until recently, the decisions related to credit loans were based on individual perceptions and the human capacity to assess the risk. The growing demand for credit has led to the use of a statistician method, known as Credit Scoring, to decide whether to or not to grant credit.
This method is widely used for consumer loans, and it is getting more used for commercial loans. The credit score is a binary classification task of basic finance. An advantage of the credit score method is the reduction of the costs of credit analysis: faster credit decisions, greater control and reduced potential risks.

\section{B. ACAD Data Preparation}

The database contains 690 cases, divided into two classes, 307 applicants "accepted" and 383 applicants "rejected". Each applicant has 15 features, including 6 nominal, 8 numeric attributes and the last one is the label of each class (accepted or rejected). This dataset is interesting because there is a good mix of attributes: continuous and nominal, nominal ratings with small and large values. Another important characteristic is that few values are missing.

Normally the classifiers based on neural networks produce better results if the training sets are balanced, presenting the same number of patterns belonging to each one of the possible classes. Considering this an adaptation of the data set is needed so some accepted patterns have been repeated instead of eliminating rejected patterns to avoid a potential loss of information.

Depending on the concrete inputs used for training and for performance evaluation it is possible to have a numerical influence on the results. To obtain results statistically independent of the distribution of the patterns a 10 fold cross validation evaluation method has been considered. Using this method the possible dependence of the results with the distribution of the samples in the training or performance evaluation sets is eliminated: all the samples are used to train the networks and all the samples are used to evaluate the performance of the results. This is applied to different executions of the experiment for the same initial neural networks, and finally mean values are calculated to establish the final performance results.

For this experiment we have created ten data sets from the ACAD with the following distribution of patterns:

- G1: 78 total patterns: 39 rejected and 39 accepted

- G2: 78 total patterns: 39 rejected and 39 accepted

- G3: 78 total patterns: 39 rejected and 39 accepted

- G4: 76 total patterns: 38 rejected and 38 accepted

- G5: 76 total patterns: 38 rejected and 38 accepted

- G6: 76 total patterns: 38 rejected and 38 accepted

- G7: 76 total patterns: 38 rejected and 38 accepted

- G8: 76 total patterns: 38 rejected and 38 accepted

- G9: 76 total patterns: 38 rejected and 38 accepted

- G10: 76 total patterns: 38 rejected and 38 accepted

Using these 10 initial sets we will create 10 different data groups. The training data groups that will be used as inputs to the networks for training the system and evaluating the evolution of the error will consist in the union of 9 of the previous 10 sets. The final evaluation that calculates the performance of the performance of the network will use the other initial set. The 10 folders will be created with the variation of the initial set that is used for evaluation and 
not for training. The networks are trained from the same initial aleatory weights presenting the data corresponding to each of the 10 final folders created. Finally the mean values of the results will be calculated to eliminate the statistical influence of the fixed selection of some patterns for training and evaluation.

\section{Koniocortex-Like Network Model}

KLN model is composed by rate code neurons whose outputs $O_{j}$ are limited between the values 0 and 1 . These values represent the probability of occurrence of an action potential. Considering the normalized input pattern $\vec{i}=\vec{I} /\|\vec{I}\|$ (lower case notation meaning vector normalization) as the external inputs to neuron $\mathrm{j}$. Normalization is performed with the $l_{1}$-norm in which:

$$
\|\vec{I}\|=\sum_{i=1}^{n}\left|I_{i}\right|
$$

And the neuron's $\mathbf{j}$ weights as the components of a vector prototype $\overrightarrow{T^{j}}$, so that $\overrightarrow{T^{j}}=\overrightarrow{W^{j}}=\left[W_{j 1}, W_{j 2}, \ldots, W_{j n}\right]$. The inner product of weights and the input pattern, the net-input of neuron $\mathrm{j}$ is calculated as net ${ }_{j}=\left\|\overrightarrow{W^{j}} \cdot \vec{i}\right\|=\| \overrightarrow{T^{j}}$ $\vec{i}\|=\| \overrightarrow{T_{\vec{I}}^{j}} \|$, the modulus of the projection of prototype $\overrightarrow{T^{j}}$ over input pattern $\vec{I}$.

The weights are modified as a consequence of the training method using the incremental version of the pre-synaptic rule:

$$
\triangle \omega=\xi I(O-\omega)
$$

Where $O$ and $I$ are the post-synaptic and pre-synaptic action potential probabilities, respectively, and $\xi$, a learning factor.

This rule is based on the plasticity curves [14] that relate the post-synaptic voltage and the weights. This curve is also influence by metaplasticity, an homeostatic neural property that changes the elongation of the plasticity curves depending on the initial synaptic weights [15], [16].

There exists a relation between the net-input of neuron $O^{j}$ and its firing probability $O_{j}$, using a conventional sigmoidal activation function.

$$
O_{j}=\frac{1}{1+e^{-k\left(n e t_{j}+0.5-2 s^{j}\right)}}
$$

Where $k$ is a curve-compressing factor and $s^{j}$ the horizontal shift of the activation function ranging from zero to one, $0<s^{j}<1$. Real neuron exhibits intrinsic plasticity [17], [18] as shown in Fig. 1, the homeostatic property that makes very active neurons to be moderated and inactive neurons to increment its firing rate. The activation function shifts leftwards or rightwards regulating the activation of scarcely or highly activated neurons, respectively.

Parameter $s^{j}$ is mathematically incorporated to the model in the neuron's activation function $f()$ relating the net-input of the neuron to its spiking probability $O_{j}$ :

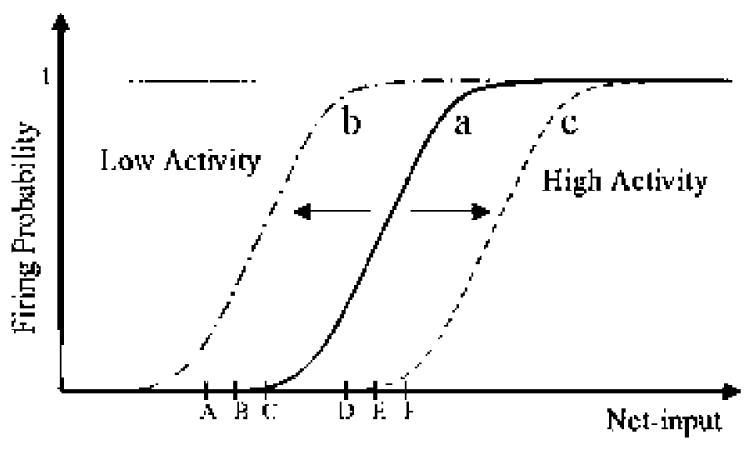

Fig. 1. Intrinsic plasticity allows the neurons' activation function to shift horizontally so that the activation function "follows" the average net-input of the neuron. (a) Initial position of the sigmoidal activation function. (b) In the case of a low regime of net-input values (as in A, B and C), intrinsic plasticity shifts the sigmoid leftwards. (c) In the case of a high regime of net-input values (as in D, E and F), intrinsic plasticity shifts the sigmoid rightwards increasing the sensitivity of the neuron.

$$
O^{j}=f\left(\left\|\overrightarrow{T_{\vec{I}}^{j}}\right\|, s^{j}\right)
$$

The following equation calculates the shift of the activation function, $s$ at time $t$ in terms of the shift and output probability of the neuron at time $t-1$.

$$
s_{t}^{j}=\frac{v \cdot O_{t-1}+s_{t-1}^{j}}{v+1}
$$

Where $v$ is the shifting velocity parameter. It is a small arbitrary factor for adjusting the shifting rate of the activation function.

Fig. 2 is the complete version of the KLN model used in the experiment. In the KLN structure, $B$ labeled neurons are inhibitory neurons including intrinsic plasticity capabilities. $S$ labeled neurons are the main neurons engaged in competition and also present intrinsic plasticity. Since each $S$ contacts a single $B$, intrinsic plasticity is regulated in both types of neurons. So if $S$ is highly activated it is the same for associated $B$. This implies that $S$ reduces its excitability and $B$ the inhibitory field surrounding $S$, affecting the final activated neuron in future classification performances. $T C$ neurons can use intrinsic plasticity to remove the mean of a series of input values. When removing the average value, patterns become more uncorrelated and easier to classify.

Fig. 2 shows that each $S$ neuron has a recurrent connection on itself that was initially intended for allowing a sustained activation over time in simple rate-code neurons. Recurrent connections are extremely rare in real neurons. Despite of this, this kind of recurrent connection was indeed present in the koniocortex. Finally $S B$ neuron is incorporated to the model to be used in pattern normalization. Similarly to real shunting/dividing inter-neurons, $S B$ neurons perform the arithmetical summation of its inputs ( $T C$ outputs), dividing the activation of its target neurons (the $S$ neurons) by this quantity. 


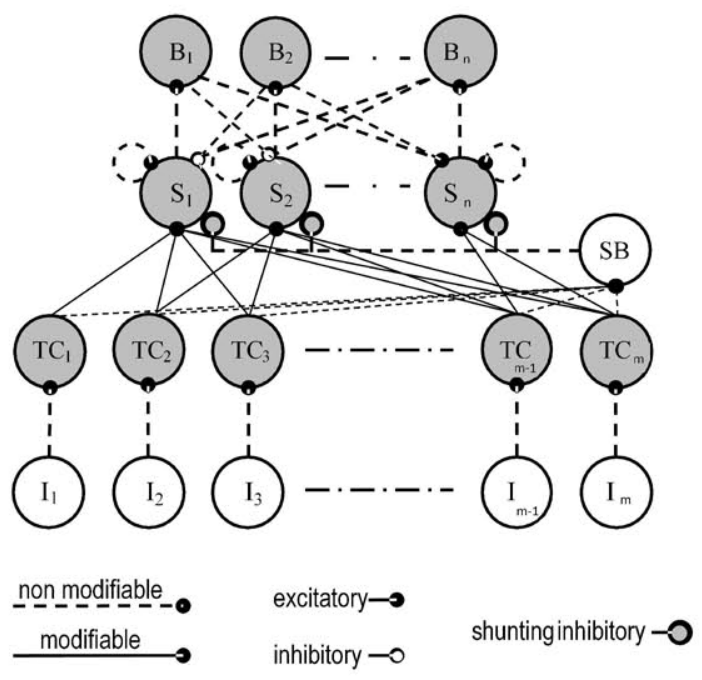

Fig. 2. Architecture of KLN applied to the classification of ACAD patterns.

\section{Network Characteristics}

In this experiment 50 different initial networks have been trained, all the models used in this study were trained and tested with the same data and validated using the 10-folder cross-validation.

Considering the concrete experiment characteristics:

1) Structure of the network:

- Number of inputs: This KLN has 14 neurons in its input layer corresponding to the number of elements that form each input pattern.

- Number of $T C$ neurons: There are 14 neurons in the $T C$ layer (similar number as input layer to be coherent with the KLN structure).

- Number of $S$ neurons: There are 2 neurons in the $S$ layer as two classes are considered in the experiment.

- Number of $B$ neurons: There 2 neurons in the upper $B$ layer associated to the $S$ output neurons.

- Activation function: Sigmoidal according to (3) and (5).

2) Conditions considered to finalize the network training:

- Reach a defined number of epochs in the network training, the number of epochs will vary during the experiment.

When an input is presented to the network and the processing is complete, one of the output neurons will present a higher activation value that the other, which output will be inhibited by the winning node. The classification of the pattern will be made considering the comparison between the output values, and the performance of the network will be evaluated in comparison with the ideal output of the network.

This KLN prototype has demonstrated to be extremely sensitive to the concrete values of the training mathematical parameters, so very small changes from the used values cause big differences in the results, even in some cases lead to a non convergence of the learning algorithm. In this case the values obtained for the parameters have been obtained using a Montecarlo approach with many simulations until adequate results have been obtained. The final values used in this simulation are $v=0.025, \xi=0.001$, compression factor for the curve $k=20.13$, the initial sigmoid shift $s=0.5$, initial weights from $T C$ to $S$ neurons are negligible and random, and non-modifiable weights are set to $W_{S_{S}}=0.85, W_{S_{B}}=0.98$, $W_{I_{T C}}=1.0$ and $W_{B_{S}}=0.5$.

\section{E. Evaluation Method}

50 different networks have been trained using the 10 fold cross validation method. Using 50 different initial networks and calculating mean values we assure that the results are independent of the initial random values in the creation of the networks. From the results obtained for the same network with each one of the folders the mean confusion matrix is obtained for each network. Once we have these 50 mean values an additional calculation is made and the final mean value is obtained as the final result of the experiment.

The following hypothesis are defined to build a confusion matrix model (as presented in table I:

- True Positive (TP) $H(1 / 1)$ : The pattern is accepted and has been classified as accepted.

- False Positive (FP) $H(1 / 0)$ : The pattern is rejected and has been classified as accepted.

- False Negative (FN) $H(0 / 1)$ : The pattern is accepted and has been classified as rejected.

- True Negative (TN) $H(0 / 0)$ : The pattern is rejected and has been classified as rejected.

TABLE I

CONFUSSION MATRIX MODEL

\begin{tabular}{|c|l|}
\hline True Positive $H(1 / 1)$ & False Positive $H(1 / 0)$ \\
\hline False Negative $H(0 / 1)$ & True Negative $H(0 / 0)$ \\
\hline
\end{tabular}

To evaluate the performance two measures are used and defined as: Sensitivity $(\boldsymbol{S E})=\mathrm{TP} /(\mathrm{TP}+\mathrm{FN})(\%)$ that evaluates the performance of the network identifying the accepted patterns, and Accuracy $(\boldsymbol{A C})=(\mathrm{TP}+\mathrm{TN}) /(\mathrm{TP}+\mathrm{TN}+\mathrm{FP}+\mathrm{FN})(\%)$ that evaluates the performance of the network classifying both kinds of patterns. TP, TN, FP, and FN stand for true positive, true negative, false positive and false negative, respectively.

We have considered sensitivity as the driver figure in these experiments.

\section{RESULTS}

\section{A. ACAD Patterns Classification}

Several variations of the experiment have been performed with different number of epochs in each experiment (considering one epoch like presenting the full set of input patterns once to the network). The output of the network is integrated by two neurons, depending on which one presents the higher level at the output we have considered that one of the classes 
TABLE II

SENSITIVITY AND ACCURACY EVOLUTION DEPENDING ON THE NUMBER OF EPOCHS

\begin{tabular}{|c|c|c|}
\hline Epochs & Sensitivity & Accuracy \\
\hline 1 & $66.00 \%$ & $63.72 \%$ \\
\hline 5 & $84.68 \%$ & $81.07 \%$ \\
\hline 8 & $87.13 \%$ & $82.19 \%$ \\
\hline 10 & $87.15 \%$ & $82.68 \%$ \\
\hline 12 & $87.14 \%$ & $82.63 \%$ \\
\hline 13 & $87.56 \%$ & $82.84 \%$ \\
\hline 14 & $87.66 \%$ & $83.10 \%$ \\
\hline 15 & $87.65 \%$ & $82.87 \%$ \\
\hline 16 & $87.33 \%$ & $83.06 \%$ \\
\hline 17 & $87.32 \%$ & $83.04 \%$ \\
\hline 18 & $86.98 \%$ & $83.02 \%$ \\
\hline 20 & $86.93 \%$ & $83.47 \%$ \\
\hline 25 & $86.89 \%$ & $83.35 \%$ \\
\hline 50 & $86.25 \%$ & $83.32 \%$ \\
\hline 75 & $85.81 \%$ & $82.85 \%$ \\
\hline 100 & $86.06 \%$ & $82.15 \%$ \\
\hline 250 & $84.49 \%$ & $81.49 \%$ \\
\hline 500 & $84.41 \%$ & $82.51 \%$ \\
\hline 1000 & $84.34 \%$ & $82.60 \%$ \\
\hline 1500 & $84.21 \%$ & $82.68 \%$ \\
\hline 2000 & $83.85 \%$ & $82.70 \%$ \\
\hline
\end{tabular}

(accepted or rejected sample) is selected by the network. Table II presents the results obtained.

It can be seen that the results are quite similar for the different number of epochs. The best results obtained correspond to 14 epochs where a high percentage of accepted patterns are correctly recognized with a good value for the general accuracy figure. The associated classification results are:

- Accuracy $=83.10 \%$

- Sensitivity $=87.66 \%$

Confusion matrix for this variation of the experiment is shown in table III.

TABLE III

CONFUSION MATRIX 14 EPOCHS

\begin{tabular}{|l|l|}
\hline $87.66 \%$ & $21.46 \%$ \\
\hline $12.34 \%$ & $78.54 \%$ \\
\hline
\end{tabular}

\section{B. Discussion of the Results}

- This experiment related to the use of KLN with real data shows that even the system is very sensitive to the concrete values of the network parameters it is able to obtain good classifications results from the data set.

- Even with a small amount of epochs the system is able to start learning and the results don't present big variations neither from the sensitivity nor for the accuracy values once the network has been able to obtain the linear independent information from the data set. Once that this situation is reached it is not possible to improve the results with a higher number of epochs in the training.

- Comparing the KLN unsupervised results, they are worse than the results of advanced supervised methods as those presented in section [19], but with differences under 5\% in the worst situation so KLN is considered to perform successful classification task without the need of previous information about the ideal result of the each pattern of the data set.

\section{State of the Art Study}

This section present a comparison of the results obtained with the KLN structure with the results obtained by other authors using different methods over the same ACAD dataset. Table IV includes the name of the researchers, the algorithm or method used in the study and the values for sensitivity (if available) and accuracy obtained.

TABLE IV

STATE OF THE ART STUDY FOR ACAD CLASSIFICATION

\begin{tabular}{|c|c|c|c|}
\hline Author (Year) & Method & Sensitivity & Accuracy \\
\hline This study & KLN & $\mathbf{8 7 . 6 6 \%}$ & $\mathbf{8 3 . 1 0 \%}$ \\
\hline West (2000) [20] & MOE & $86.70 \%$ & $86.68 \%$ \\
\hline Ong (2005) [21] & GP & - & $88.27 \%$ \\
\hline Huang (2005) [22] & 2SGP & - & $89.17 \%$ \\
\hline Martens (2007) [23] & SVM & - & $85.70 \%$ \\
\hline Hoffmann (2007) [24] & Bayes & - & $86.70 \%$ \\
\hline Huang (2007) [25] & GA-SVM & - & $86.90 \%$ \\
\hline Peng (2008) [26] & MCQP & $87.00 \%$ & $86.38 \%$ \\
\hline Tsai (2008) [27] & Multi-Classifiers & - & $87.25 \%$ \\
\hline Nanni (2009) [28] & LMNC & - & $87.44 \%$ \\
\hline Xu (2009) [29] & HARA & - & $89.28 \%$ \\
\hline Luo (2009) [30] & CLC & - & $86.52 \%$ \\
\hline Tsai (2009) [31] & PCA & - & $89.93 \%$ \\
\hline Ping (2009) [32] & RBF-SVM & - & $87.52 \%$ \\
\hline Chen (2009) [33] & LDA+SVN & - & $86.52 \%$ \\
\hline Wang (2011) [34] & Boosting & - & $86.56 \%$ \\
\hline Wang (2012) [35] & RS-Bagging DT & - & $88.17 \%$ \\
\hline Vukovic (2012) [36] & Pure CBRPFGA & - & $88.12 \%$ \\
\hline Tsai (2014) [37] & SOM + MLP & - & $91.61 \%$ \\
\hline Semenkina (2014) [38] & SelfCEA & - & $90.22 \%$ \\
\hline Zhao (2015) [39] & MLP Scoring & - & $87.00 \%$ \\
\hline Liang (2015) [40] & Naive Bayes & - & $86.09 \%$ \\
\hline Xiao (2016) [41] & ECSC & - & $87.62 \%$ \\
\hline Chen (2016) [42] & PBIL-AIS & - & $86.23 \%$ \\
\hline
\end{tabular}

\section{CONCLuSions}

In this work we have presented the application of the theoretical basis of the Koniocortex-Like Network to a real complex data set. It is observed that the results are very similar to the ones obtained in previous supervised training experiments. The figures obtained for accuracy and sensitivity are almost the same with independence of the number of iterations used in the unsupervised training, so it seems that the linear independent information present in the data is identified and processed with a very small number of iterations which allows to reduce the computational load of the method. KLN model is still in a non optimized prototyping phase, but even in this situation the system is capable of obtaining accurate results able to compete with many state-of-the-art ANN models. These model is based in different plasticity concepts that can be widely applied to different areas inside BI to improve the BD extraction and classification methods. 


\section{REFERENCES}

[1] K. D. Miller, "Synaptic economics: Competition and cooperation in synaptic plasticity", Neuron, vol. 17, pp. 371-374, 1996.

[2] D. Andina, A. Alvarez-Vellisco, A. Jevtic and J. Fombellida, "Artificial metaplasticity can improve artificial neural network learning", Intelligent Automation and Soft Computing Special Issue in Signal Processing and Soft Computing, Vol. 15(4), pp. 681-694, 2009.

[3] D. Andina D and J. Ropero-Pelaez, "On the biological plausibility of artificial metaplasticity learning algorithm", Neurocomputing, 2012.

[4] J. Ropero-Peláez and D. Andina, "Do biological synapses perform probabilistic computations?", Neurocomputing, 2012.

[5] J. Ropero-Peláez and D. Andina, "The Koniocortex-Like Network: a new biologically plausible unsupervised neural network", Artificial Computation in Biology and Medicine, Vol. 9107, pp. 163-174, 2015.

[6] J. Ropero-Peláez, M. A. Aguiar-Furucho M.A. and D. Andina, "Intrinsic Plasticity for Natural Competition in Koniocortex-Like Neural Networks", Int J Neural Syst, Vol. 16(5), 2016.

[7] J. Fombellida, J. Ropero-Peláez and D. Andina, "Koniocortex-Like Network Unsupervised Learning Surpasses Supervised Results on WBCD Breast Cancer Database", International Work-Conference on the Interplay Between Natural and Artificial Computation, IWINAC 2017 Proceedings Part II, pp. 32-41, 2017.

[8] http://archive.ics.uci.edu/ml/datasets.html/

[9] A. Groznik, "Towards multi utility management in Europe", ICEE 2014: International Conference on Energy and Environment, Vol. 2(8), pp. 195, 2014.

[10] J. Cardenas, L. Gemoets, J. Rosas and R. Sarfi, "A literature survey on Smart Grid distribution: an analytical approach", Journal of Cleaner Production, Vol. 65, pp. 202-216, 2014.

[11] T. Singer, "Information Engineering: The search for business intelligence", Plant Engineering, pp. 34-36, 2001.

[12] E. Rubin, A. Rubin, "The impact of Business Intelligence systems on stock return volatility", Information and Management, Vol. 50, pp. 67$75,2013$.

[13] M. Kezunovic, V. Vittal, S. Meliopoulos, T. Mount, "The Big Picture: Smart Research for Large-Scale Integrated Smart Grid Solutions", Power and Energy Magazine IEEE, Vol. 10(4), pp. 22-34, 2014.

[14] A. Artola, S. Brocher and W. Singer, "Different voltage-dependent threshold for inducing long-term depression and long-term potentiation in slices of rat visual córtex", Nature, Vol. 347, pp. 69-72, 1990.

[15] W. C. Abraham and M. F Bear, "Metaplasticity: the plasticity of synaptic plasticity", Trends in Neuroscience, vol. 19, pp. 126-130, 1996.

[16] W. C. Abraham and W. P. Tate, "Metaplasticity: a new vista across the field of synaptic plasticity", Progress in Neurobiology, vol. 52, pp. 303-323, 1997.

[17] N. S. Desai, "Homeostatic plasticity in the CNS: synaptic and intrinsic forms", Journal of Physiology, Vol. 97(4-6), pp. 391-402, 2003.

[18] N. S. Desai, L. C. Rutherford and G. G. Turrigiano, "Plasticity in the intrinsic excitability of cortical pyramidal neurons", Nature Neurosciences, Vol. 2, pp. 515-520, 1999.

[19] J. Fombellida, I. Martín-Rubio, S. Torres-Alegre and D. Andina, "Tackling Business Intelligence with Bioinspired Deep Learning", Neural Computing and Applications, in press.

[20] D West, "Neural network credit scoring models", Computers and Operations Research, Vol. 27,pp. 1131-1152, 2000.

[21] C. Ong, J. J. Huang and G. H. Tzeng, "Building credit scoring models using genetic programming", Expert Systems with Applications, Vol. 29, pp. 41-47, 2005.

[22] J. J. Huang, G. H. Tzeng and C. S. Ong, "Two-stage genetic programming (2SGP) for the credit scoring model", Applied Mathematics and Computation, Vol, 174(2), pp. 1039-1053, 2005.

[23] D. Martens, B. Baesens, T. Van Gestel and J. Vanthienen, "Comprehensible credit scoring models using rule extraction from support vector machines", European Journal of Operational Research, Vol. 183(3), pp $1466-1476,2007$.

[24] F. Hoffmann, B. Baesens, C. Mues, T. Van Gestel and J. Vanthienen "Inferring descriptive and approximate fuzzy rules for credit scoring using evolutionary algorithms", European Journal of Operational Research, Vol. 177, pp. 540-556, 2007.

[25] C. L. Huang, M. C. Chen and C. J. Wang, "Credit scoring with a data mining approach based on support vector machines", Expert Systems with Applications, Vol. 33(4), pp. 847-856, 2007.
[26] Y. Peng, G. Kou, Y. Shi and Z. Chen, "A Multi-criteria Convex Quadratic Programming model for credit data analysis", Decision Support Systems, Vol. 44(4), pp. 1016-1030, 2008

[27] C. F. Tsai and J. W. Z. Wu, "Using neural network ensembles for bankruptcy prediction and credit scoring", Expert Systems with Applications, Vol. 44(4), pp. 2639-2649, 2008.

[28] L. Nanni and A. Lumini, "An experimental comparison of ensemble of classifiers for bankruptcy prediction and credit scoring", Expert Systems with Applications, Vol. 36, pp. 3028-3033, 2009.

[29] X. Xu, C. Zhou and Z, Wang, "Credit scoring algorithm based on link analysis ranking with support vector machine", Expert Systems with Applications, Vol. 36(2), pp. 2625-2632, 2009.

[30] S. T. Luo, B. W. Cheng and C. H. Hsieh, "Prediction model building with clustering-launched classification and support vector machines in credit scoring", Expert Systems with Applications, Vol. 36(4), pp. 7562-7566, 2009.

[31] C. F. Tsai, "Feature selection in bankruptcy prediction", KnowledgeBased Systems, Vol. 22(22), pp.120-127, 2009.

[32] Y. Ping, "Hybrid Classifier Using Neighborhood Rough Set and SVM for Credit Scoring", In Proc. BIFE 09, International Conference on Business Intelligence and Financial Engineering., pp. 138-142, 2009.

[33] F. L. Chen and F. C. Li, "Combination of feature selection approaches with SVM in credit scoring", Expert Systems with Applications, Vol. 37(7), pp. 4902-4909, 2009.

[34] G. Wang, J. Hao, J. Ma and H. Kiang, "A comparative assessment of ensemble learning for credit scoring", Expert Systems with Applications, Vol. 38(1), pp. 223-230, 2011.

[35] G. Wang, J. Ma, L. Huang and K. Xu, "Two credit scoring models based on dual strategy ensemble trees", Knowledge-Based Systems, Vol. 26, pp. 61-68, 2012.

[36] S. Vukovic, B. Delibasic, A. Uzelac and M. Suknovic, "A casebased reasoning model that uses preference theory functions for credit scoring", Expert Systems with Applications, Vol. 39(9), pp. 8389-8395, 2012.

[37] C. F. Tsai, "Combining cluster analysis with classifier ensembles to predict financial distress", Information Fusion, Vol. 16, pp.46-58, 2014

[38] M. Semenkina and E. Semenkin, "Hybrid Self-configuring Evolutionary Algorithm for Automated Design of Fuzzy Classifier", International Conference in Swarm Intelligence ICSI 2014, pp. 310-317, 2014.

[39] Z. Zhao, S. Xu, B. H. Kang, M. M. J. Kabir, Y. Liu and R. Wasinger, "Investigation and improvement of multi-layer perceptron neural networks for credit scoring", Expert Systems with Applications, Vol. 42, pp. 3508-3516, 2015.

[40] D. Liang, C. F. Tsai and H. T. Wu, "The effect of feature selection on financial distress prediction", Knowledge-Based Systems, Vol. 73, pp. 289-297, 2015.

[41] H. Xiao, Z. Xiao, and Y. Wang, "Ensemble classification based on supervised clustering for credit scoring", Applied Soft Computing, Vol. 437, pp. 73-86, 2016.

[42] M. H. Chen, P. C. Chang and J. L. Wu, "A population-based incremental learning approach with artificial immune system for network intrusion detection", Engineering Applications of Artificial Intelligence, Vol. 51, pp. 171-181, 2016 